\title{
Clinical outcomes of patients with acute lymphoblastic leukemia receiving the hyper-CVAD regimen and assessment of the risk of hepatitis flares due to hepatitis $B$ virus reactivation after chemotherapy
}

Man Fai Law ${ }^{1,2}$, Hay Nun Chan ${ }^{1}$, Shun Yin Kong ${ }^{1}$, Ho Kei Lai ${ }^{1}$, Chung Yin Ha ${ }^{1}$, Celia Ng1, Yiu Ming Yeung ${ }^{1}$, Sze Fai Yip ${ }^{1}$

\author{
${ }^{1}$ Department of Medicine, Tuen Mun Hospital, Hong Kong, Hong Kong \\ 2Department of Medicine and Therapeutics, Prince of Wales Hospital, Hong Kong, \\ Hong Kong
}

Submitted: 13 July 2018; Accepted: 2 February 2019 Online publication: 19 March 2021

Arch Med Sci 2022; 18 (1): 121-128

DOI: https://doi.org/10.5114/aoms/103606

Copyright (c) 2021 Termedia \& Banach

\begin{abstract}
Introduction: Hyper-CVAD (hyperfractionated cyclophosphamide, vincristine, doxorubicin and dexamethasone) has become a popular regimen for adults with acute lymphoblastic leukemia (ALL). We assessed the efficacy and tolerability of hyper-CVAD in the treatment of adult ALL.

Material and methods: We retrospectively reviewed ALL patients aged 18 or above receiving the hyper-CVAD regimen. We assessed complete remission rate and overall survival, as well as hepatitis $B$ carrier rate and hepatitis flare due to hepatitis $B$ virus (HBV) reactivation.

Results: Fifty-two patients were treated with the hyper-CVAD regimen. The median age at diagnosis was 42 years; $27 \%$ of patients were Philadelphia $(\mathrm{Ph})$ chromosome positive. The complete remission (CR) rate was $90.4 \%$ after the first cycle of chemotherapy. The induction mortality rate was $1.9 \%$. Three patients required two cycles of hyper-CVAD to achieve CR. The median overall survival was 39.6 months and the 5 -year overall survival was $50 \%$. Age over 30 years and white blood cell count of more than $30 \times 10^{9} / 1$ were found to be prognostic for poor overall survival in multivariate analysis. The hepatitis B carrier rate was $17 \%$ in our cohort, and the rate of hepatitis flare due to HBV reactivation was $11 \%$ in patients with current infection.

Conclusions: Hyper-CVAD is feasible and tolerable with a good CR rate in the treatment of adult ALL patients. It is an option for the treatment of ALL. Antiviral prophylaxis should be considered in ALL patients with HBV infection to reduce the risk of HBV reactivation.
\end{abstract}

Key words: acute lymphoblastic leukemia, hepatitis B virus reactivation, hyper-CVAD.

\section{Introduction}

Many induction regimens have been developed for the treatment of adult patients with acute lymphoblastic leukemia (ALL). The hyper-CVAD regimen (hyperfractionated cyclophosphamide, vincristine, doxorubicin and dexamethasone) has become a popular therapy for adult ALL patients. An effective dose-intensive regimen for adult patients with ALL was developed at the M.D. Anderson Cancer Center in the United States,
Corresponding author:

Man Fai Law

Department of Medicine and Therapeutics

Prince of Wales

Hospital

Hong Kong

Phone: 852-97763090

E-mail: mflaw99@yahoo.

com.hk 
and involves alternating hyper-CVAD with a highdose MTX-Ara-C (methotrexate and cytarabine) regimen [1]. The treatment response to this regimen was good, with a complete remission rate of $92 \%$ and an estimated 5 -year overall survival of $38 \%$ [2].

Although the remission rates with induction chemotherapy are good, most adult patients with ALL will finally relapse. Hemopoietic stem cell transplant (HSCT) is often performed to reduce the risk of relapse, especially in patients with high-risk ALL.

Although hyper-CVAD is generally associated with predictable and manageable toxicities, this regimen has the potential to reactivate viral infections such as hepatitis B virus (HBV) in chronically infected patients. Globally about 350 million people have chronic infection with HBV. The course of $\mathrm{HBV}$ reactivation in cancer patients is variable and may range from asymptomatic to fulminant hepatitis and even death. Fatal cases of HBV reactivation have been reported in patients with hematological malignancies including lymphoma and leukemia [3-6]. Hepatitis flare can also cause interruption of chemotherapy or a dose reduction, which may affect the efficacy of the chemotherapy. Clinicians initiating chemotherapy in hematology patients should be aware of the potential for hepatitis flare, which is a severe but preventable complication. There is limited published information on the carrier rate of hepatitis $B$ in $A L L$ and hepatitis $B$ flare due to HBV reactivation in ALL patients. It is especially an important issue in HBV endemic areas, such as Asia.

We studied the outcomes of ALL patients treated with the hyper-CVAD regimen. We also investigated the carrier rate of HBV and risk of hepatitis flare due to HBV reactivation after chemotherapy. The results from our study will add information to the literature on these two issues.

\section{Material and methods}

\section{Patient selection}

This was a retrospective analysis of clinical records for patients aged 18 or above, with untreated ALL who had received hyper-CVAD chemotherapy between January 2005 and December 2016 in Tuen Mun Hospital in Hong Kong. Baseline parameters such as age at diagnosis, sex, clinical presentations of leukemia, central nervous system (CNS) involvement at diagnosis, the presence of any large mediastinal masses, complete blood profile and bone marrow results were collected.

ALL diagnosis was confirmed using bone marrow aspirate and trephine morphology, immunophenotype, and cytogenetic results. Patients were referred for allogenic stem cell transplant if a suitable donor was found and the disease was in remission. The complete remission rate, overall survival and toxicity of the hyper-CVAD regimen were analyzed.

Viral screening of blood samples included hepatitis B surface antigen ( $\mathrm{HBsAg}$ ) and hepatitis B surface antibody (anti-HBs) as well as hepatitis C virus antibody (anti-HCV) and human immunodeficiency virus (HIV). Liver biochemistry was monitored regularly before and after starting chemotherapy. Before 2010, we did not routinely screen for the presence of antibody to hepatitis B core antigen (anti-HBc) in $\mathrm{ALL}$ patients, but anti-HBC testing was added to the screening protocol from 2010 onwards after the report of HBV reactivation in patients with a hematological malignancy and anti-HBc [7].

We defined the HBV-infected patients as those with detectable levels of HBsAg when ALL was diagnosed. Hepatitis flare due to HBV reactivation was defined as a 10-fold rise in HBV deoxyribonucleic acid (HBV-DNA) and 3-fold rise in serum alanine aminotransferase (ALT) compared with baseline. For anti-HBc positive patients, HBV reactivation was defined as seroconversion from HBV DNA negative to positive.

In patients who developed hepatitis, the severity was classified according to the criteria of the World Health Organization (WHO). Grade 1 was defined as ALT elevated to $>1.25 \times$ the upper limit of normal (ULN) and up to $2.5 \times$ ULN; grade 2 was ALT elevated to > 2.5 $\times$ ULN and up to $5.0 \times$ ULN; grade 3 was an elevation of ALT > 5.0× ULN and up to $10.0 \times$ ULN; and grade 4 was an elevation of $\mathrm{ALT}>10 \times$ ULN [8].

\section{Treatment}

The chemotherapy regimen included a dose-intensive phase in which cycles of hyper-CVAD were alternated with high-dose intravenous methotrexate (MTX) and cytarabine, up to a maximum of 8 cycles, followed by 2 years of maintenance therapy for patients not undergoing HSCT. The details of the protocol have been described previously $[1,2]$.

Patients with Philadelphia chromosome (Ph) positive ALL also received a tyrosine kinase inhibitor concurrently with hyper-CVAD chemotherapy during induction, consolidation and maintenance therapy. Patients with high-risk disease (complex cytogenetics, high initial white blood cell (WBC) count) and a matched donor underwent allogenic HSCT. If there was no matched donor, they received maintenance therapy for 2 years. Individual patients who had a matched family donor but did not have high-risk features were also considered for HSCT.

CNS prophylaxis consisted of intrathecal administrations of MTX $12 \mathrm{mg}$ on day 2 and cy- 
tarabine $100 \mathrm{mg}$ on day 7 of each cycle. All patients received standard antimicrobial prophylaxis consisting of oral levofloxacin, itraconazole and acyclovir starting from the beginning of chemotherapy. They also received trimethoprim/sulfamethoxazole for prevention of Pneumocystis jiroveci pneumonia. Patients who were positive for $\mathrm{HBsAg}$ or anti-HBc were given specific antiviral prophylaxis for HBV reactivation from the beginning of chemotherapy until at least 1 year after stopping chemotherapy. The antiviral prophylaxis was lamivudine $100 \mathrm{mg}$ daily from 2005 to 2013, but was changed to entecavir $0.5 \mathrm{mg}$ daily in 2014 because of growing evidence that entecavir was a more effective option than lamivudine for preventing HBV reactivation [9].

\section{Response assessment and statistical analysis}

We performed bone marrow examination at the end of the induction phase of chemotherapy. If complete remission (CR) could not be achieved, bone marrow examination was repeated at the end of each cycle of therapy until CR was documented. CR was defined as less than $5 \%$ blasts on bone marrow morphologic examination as well as hematologic recovery.

The primary outcomes were overall survival and $C R$ rate. A univariate analysis of risk factors for decreased overall survival or death was performed by calculating the hazard ratio (HR) and 95\% confidence intervals $(\mathrm{Cl})$. The prognostic significance of age, sex, WBC, Ph chromosome, HBV status and bulky disease was assessed. This was followed by a Cox proportional hazards model using variables with a significant impact on outcomes in the univariate analysis $(p<0.05)$. We calculated the overall survival from the date of ALL diagnosis until death or censored at the last follow-up date, and analyzed using Kaplan-Meier survival analysis and the logrank test. The $p$-values were 2 -sided and $p$-values of $<0.05$ were defined as statistically significant.

\section{Results}

\section{Patient characteristics}

A total of $52 \mathrm{ALL}$ patients were treated with hyper-CVAD in the study period (Table I). The median age at diagnosis was 42 years (range: $20-74$ ). The male to female ratio was 1.36 (30/22). The median follow-up time was 72 months (1.0149.1). No patient had CNS disease at diagnosis. Forty-one patients had B-cell ALL (79\%). The median WBC was $11.35(0.4-178) \times 10^{9} /$, median hemoglobin was $9.6(3.5-14.6) \mathrm{g} / \mathrm{dl}$, and median platelet count was $56(7-429) \times 10^{9} /$. Thirteen $(25 \%)$ patients had a WBC more than $30 \times 10^{9}$ cells/l at diagnosis.
Diagnostic cytogenetic results were available in 51 (95\%) patients, but no bone marrow aspirate could be obtained (dry tap) in one patient. Fourteen $(27 \%)$ patients were Ph-positive, and were given tyrosine kinase inhibitor together with hyper-CVAD chemotherapy.

\section{Treatment response}

Forty-seven (90.4\%) patients achieved CR after the first cycle of induction. Three patients required two cycles of hyper-CVAD to achieve CR. One had primary refractory disease. Another patient died of infection during induction chemotherapy. The median overall survival was 39.6 months and the 5 -year survival rate was $50 \%$ (Figure 1 ). Bone marrow relapse occurred in 21 patients. The median time from CR to relapse was 9 months (3-66

Table I. Characteristics of ALL patients in our study

\begin{tabular}{|c|c|}
\hline Characteristics, $n(\%)$ & ALL patients $(n=52)$ \\
\hline \multicolumn{2}{|l|}{ Age categories: } \\
\hline $18-30$ years & $16(30.8)$ \\
\hline $31-60$ years & $32(61.5)$ \\
\hline$>60$ years & $4(7.7)$ \\
\hline \multicolumn{2}{|l|}{ Sex: } \\
\hline Male & $30(57.7)$ \\
\hline Female & $22(42.3)$ \\
\hline \multicolumn{2}{|l|}{$\begin{array}{l}\text { ALL signs and symptoms } \\
\text { at diagnosis: }\end{array}$} \\
\hline Bone or joint pain & $6(11.5)$ \\
\hline Lymphadenopathy & $5(9.6)$ \\
\hline Pleural effusion & $2(3.8)$ \\
\hline Mediastinal mass & $2(3.8)$ \\
\hline CNS disease & 0 \\
\hline \multicolumn{2}{|l|}{ Cytogenetics: } \\
\hline Ph chromosome & $14(27)$ \\
\hline Complex cytogenetics & $9(17)$ \\
\hline$t(1,19)$ & $1(2.0)$ \\
\hline Normal & $18(35)$ \\
\hline No growth & $1(2.0)$ \\
\hline \multicolumn{2}{|l|}{ WBC count categories: } \\
\hline$>30 \times 10^{9} / 1$ & $21(40.4)$ \\
\hline$<30 \times 10^{9} / 1$ & $31(59.6)$ \\
\hline \multicolumn{2}{|l|}{ Bulky disease: } \\
\hline Yes & $2(3.8)$ \\
\hline No & $50(96.2)$ \\
\hline \multicolumn{2}{|l|}{ HBsAg status: } \\
\hline Positive & $9(17.3)$ \\
\hline Negative & $43(82.7)$ \\
\hline
\end{tabular}

ALL - acute lymphoblastic leukemia, CNS - central nervous system, Ph - Philadelphia chromosome, WBC - white blood cells, HBsAghepatitis B surface antigen. 


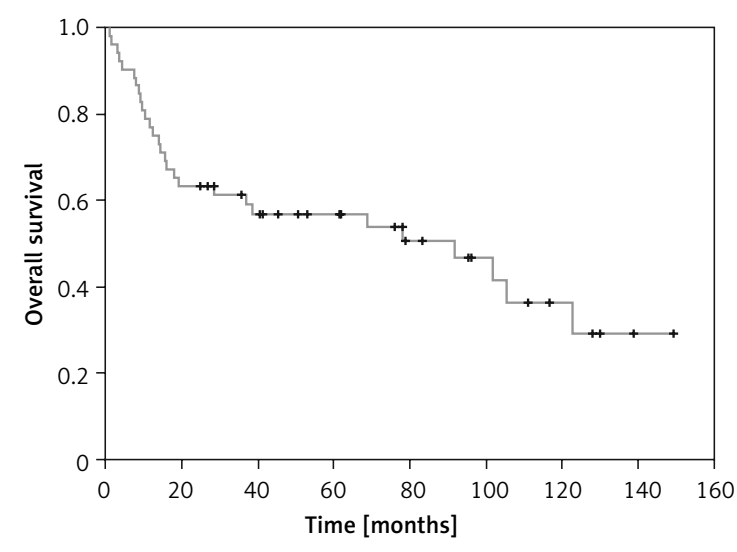

Figure 1. Overall survival of acute lymphoblastic leukemia (ALL) patients treated with hyper-CVAD (hyperfractionated cyclophosphamide, vincristine, doxorubicin and dexamethasone)

months). There was no CNS relapse. One (1.9\%) patient died during induction. This patient was 58 years old, and died of pneumonia and Escherichia coli septicemia about 1 month after his ALL was diagnosed.

Overall, 31 (59.6\%) patients underwent allogenic HSCT, of whom 14 were Ph-positive. Twelve of the 31 patients relapsed after HSCT, and only one of these patients achieved further CR after post-HSCT relapse.

\section{Prognostic factors}

Risk factors for reduced overall survival on univariate analysis included WBC >30 × $10^{9}$ cells $/ \mathrm{l}$ (hazard ratio $(H R)=2.53,95 \%$ confidence interval (Cl): 1.18-5.422, $p=0.017)$, age $>30$ years $(\mathrm{HR}=4.40,95 \% \mathrm{Cl}: 1.49-13.02, p=0.007)$, male sex $(\mathrm{HR}=2.83,95 \% \mathrm{Cl}: 1.23-6.53, p=0.015)$, and presence of $\mathrm{Ph}$ chromosome $(\mathrm{HR}=2.59$, 95\% Cl: $1.18-5.69, p=0.017)$. HBsAg status was not significantly associated with overall survival $(\mathrm{HR}=0.69,95 \% \mathrm{Cl}: 0.24-1.99, p=0.49)$ (Table II).

Table II. Risk factors for death or decreased overall survival in univariate analysis

\begin{tabular}{|lcc|}
\hline Clinical factor & Hazard ratio $(95 \% \mathrm{Cl})$ & $\boldsymbol{P}$-value \\
\hline Age $>30$ years & $4.40(1.49-13.02)$ & 0.007 \\
\hline Male sex & $2.83(1.23-6.53)$ & 0.015 \\
\hline WBC > 30 × $10^{9} / \mathrm{l}$ & $2.53(1.18-5.42)$ & 0.017 \\
\hline Ph positive & $2.59(1.18-5.69)$ & 0.017 \\
\hline HBsAg positive & $0.69(0.24-1.99)$ & 0.49 \\
\hline B-ALL & $2.52(0.59-10.63)$ & 0.21 \\
\hline B symptoms & $0.97(0.5-1.86)$ & 0.92 \\
\hline Bulky disease & $0.40(0.06-2.93)$ & 0.37 \\
\hline
\end{tabular}

WBC - white blood cells, Ph - Philadelphia chromosome, HBsAghepatitis $B$ surface antigen, ALL - acute lymphoblastic leukemia, $\mathrm{Cl}$ - confidence interval.
In the multivariate analysis using a Cox proportional model, WBC more than $30 \times 10^{9}$ cells $/ \mathrm{l}$ $(\mathrm{HR}=2.52,95 \% \mathrm{Cl}: 1.11-5.75, p=0.028)$ and age $>30$ years $(\mathrm{HR}=3.56,95 \% \mathrm{Cl}: 1.14-11.16, p=$ $0.029)$ remained significant prognostic factors for reduced overall survival.

\section{Hepatitis flares in ALL patients}

Nine $(17.3 \%)$ patients were $\mathrm{HBsAg}$ positive at the time of ALL diagnosis. Of the 28 (14\%) patients who were tested for anti-HBc, four were anti-HBc positive. These 13 patients were given antiviral prophylaxis from the beginning of chemotherapy until at least 12 months after finishing chemotherapy.

One (11\%) HBsAg-positive patient developed a hepatitis flare due to HBV reactivation., at about 12 months after starting chemotherapy. He was on lamivudine prophylaxis, and the peak HBV-DNA was $4.43 \times 10^{6} \mathrm{IU} / \mathrm{ml}$. The highest ALT level was $1061 \mathrm{U} / \mathrm{l}$. Ultrasound of the abdomen was unremarkable. Antiviral therapy was changed to entecavir after the hepatitis flare. Liver enzymes gradually normalized and the patient could continue the chemotherapy. HBV-DNA was not detected later.

\section{Toxicity}

One patient died of pneumonia during induction chemotherapy. Three patients died of infective complications (pneumonia) during hyper-CVAD chemotherapy. These patients ranged in age from 21 to 67 years. Two of these patients had relapsed leukemia.

Three patients developed perianal abscesses, but these responded to antibiotic treatment and surgical drainage of the abscess. One patient had CNS infection. None of the patients in our cohort developed thrombosis, and there was no cerebellar toxicity resulting from high-dose cytarabine.

\section{Discussion}

The hyper-CVAD regimen was developed at the M.D. Anderson Cancer Center for the treatment of ALL and high-grade lymphoma patients [10]. The outcomes of the hyper-CVAD regimen have been shown to be significantly superior to outcomes with the VAD (vincristine, doxorubicin and dexamethasone) regimen $[1,2]$, with a CR rate of $91 \%$ and a 5 -year survival rate of $39 \%$ [1, 2]. Hyper-CVAD is now one of the most frequently used chemotherapeutic regimens for the treatment of adult ALL.

Since the introduction of hyper-CVAD for ALL at our institution, 52 patients have been treated (median age: 42 years), and this retrospective analysis showed that the CR rate in this real-world cohort of ALL patients receiving hyper-CVAD was 
$90 \%$. The induction mortality rate was $1.8 \%$. The median survival was 39.6 months and the 5 -year survival rate was $50 \%$. Three patients in our study died of pneumonia. Two of them also had relapsed leukemia, which could have contributed to the mortality.

Our results compare favorably with the results of other published studies (Table III) [1, 2, 11-16]. The overall survival in our study was slightly higher than some of the previously published studies. It could be due to more HSCT in high-risk patients and adequate antibiotic prophylaxis in our study. Patients in some studies might not receive adequate anti-infective prophylaxis [13-15]. For example, trimethoprim/sulfamethoxazole prophylaxis for Pneumocystis jiroveci was not applied in all study institutions in one study [14].

In our experience, hyper-CVAD was easy to administer. Some patients could be discharged from hospital soon after finishing chemotherapy. The regimen was tolerable and the toxicity was acceptable. Compared with other studies, a higher proportion of ALL patients in our cohort underwent HSCT. HSCT is an attractive option for the treatment of ALL, especially in younger patients.

Previous analyses have shown that advanced age, high WBC count, and presence of the Ph chromosome are poor prognostic factors in ALL [17]. Results in our cohort also showed that WBC $>30$ $\times 10^{9}$ cells $/ /$ and age $>30$ years predict reduced survival after hyper-CVAD in ALL.

In addition to markers of risk such as WBC and age, there is increasing evidence that biomarkers of cellular apoptosis and autophagy, such as beclin-1 and B-cell lymphoma-2 (Bcl-2) levels, may have potential prognostic value in ALL [18]. Autophagy is a catabolic pathway involving degradation of lysosomes and then recycling of cell proteins and organelles [19]. Abdelsalam et al. studied the expression levels of a series of autophagic beclin-1, pro-apoptotic (Bad and Bax), and anti-apoptotic (Bcl-2 and $\mathrm{Bcl}-\mathrm{xL}$ ) parameters, illustrating the correlation between their levels in ALL. There was a positive correlation with $\mathrm{Bcl}-2$, while there was a negative correlation with beclin-1 [18].

\section{Ph positive ALL}

Our patients received a tyrosine kinase inhibitor (TKI) in combination with hyper-CVAD chemotherapy if they were $\mathrm{Ph}$ positive. They also received HSCT if a suitable donor was available. The incorporation of TKI into hyper-CVAD chemotherapy in the treatment of Ph-positive ALL has improved the outcome of these patients, especially when the TKIs are combined early and continuously with chemotherapy [20-22]. Therefore, TKIs in combination with chemotherapy are the standard of care for all patients with Ph-positive ALL.

Second-generation TKIs appear to be more potent than imatinib [23-25]. Dasatinib also inhibits SRC kinases, which are involved in the pathophysiology of Ph-positive ALL [26]. Hyper-CVAD in combination with dasatinib has shown encouraging results in ALL patients, with a CR rate of $96 \%$, and median overall survival of 47 months [27]. Another study comparing ponatinib with dasatinib in combination with hyper-CVAD showed an even better survival benefit in the ponatinib group. The 3 -year overall survival rates were $83 \%$ and $56 \%$ in the ponatinib and dasatinib groups, respectively [28]. Despite the improved responses with TKIs in Ph-positive ALL, allogenic HSCT remains the standard of treatment $[29,30]$.

\section{$\mathrm{HBV}$ reactivation in $\mathrm{ALL}$ patients with $\mathrm{HBV}$ infection}

The HBV carrier rate was $17.3 \%$ in our cohort, which is higher than in the general population in Hong Kong [31]. There is evidence that HBV infection is more common in patients with lymphoma $[32,33]$. It has been postulated that HBV can inte-

Table III. Comparison of results of hyper-CVAD in ALL patients in different studies

\begin{tabular}{|c|c|c|c|c|c|c|c|}
\hline Reference & $N$ & $\begin{array}{c}\text { Median } \\
\text { age } \\
\text { [years] }\end{array}$ & $\begin{array}{c}\mathrm{Ph} \\
\text { positive }\end{array}$ & CR & $\begin{array}{c}\text { Induction } \\
\text { mortality } \\
\text { rate }\end{array}$ & $\begin{array}{c}\text { Median } \\
\text { survival } \\
\text { [months] }\end{array}$ & Overall survival \\
\hline Kantarjian et al. 2000 [1] & 204 & 39.5 & $17 \%$ & $91 \%$ & $5 \%$ & 35 & $39 \%$ at 5 years \\
\hline Kantarjian et al. 2004 [2] & 288 & 40 & $17 \%$ & $92 \%$ & $5 \%$ & 32 & $38 \%$ at 5 years \\
\hline Xu et al. 2008 [11] & 53 & 30 & $11.3 \%$ & $73.6 \%$ & $0 \%$ & 48.7 & $83 \%$ at 2 years \\
\hline Morris et al. 2011 [12] & 63 & 29 & $7 \%$ & $86 \%$ & $5 \%$ & 54 & $48 \%$ at 5 years \\
\hline Abbasi et al. 2013 [13] & 66 & 32.9 & $16 \%$ & $90 \%$ & $5 \%$ & 30 & $20 \%$ at 5 years \\
\hline Buyukasik et al. 2013 [14] & 57 & 29 & $12 \%$ & $84.2 \%$ & $10.5 \%$ & 16.4 & $26.3 \%$ at 5 years \\
\hline Alacacioglu et al. 2014 [15] & 30 & 30.5 & $13.3 \%$ & $96 \%$ & N/A & 41.5 & $34 \%$ at 5 years \\
\hline Portugal et al. 2015 [16] & 49 & 30 & $13.6 \%$ & $93.8 \%$ & $4 \%$ & 24.4 & $35 \%$ at 5 years \\
\hline Present study & 52 & 42 & $27 \%$ & $90.4 \%$ & $1.8 \%$ & 39.6 & $50 \%$ at 5 years \\
\hline
\end{tabular}

Hyper-CVAD - hyperfractionated cyclophosphamide, vincristine, doxorubicin and dexamethasone, ALL - acute lymphoblastic leukemia, $N$ - number, $P h$ - Philadelphia chromosome, CR-complete remission, N/A-not available. 
grate into the host genome, where it can downregulate the expression of tumor suppression genes or increase expression of cellular oncogenes [34, 35]. Alternatively, the persistent antigenic stimulation and B-lymphocyte proliferation that occur during chronic HBV infection may predispose patients to genetic aberrations or double-stranded DNA breaks that could lead to malignant transformation and also proliferation.

Most of the data on HBV reactivation in cancer patients are from lymphoma patients being given chemotherapy and rituximab. There are few data on acute leukemia, and the correlation between HBV infection and ALL is not yet known. In a previous study in patients with acute myeloid leukemia $\mathrm{HBV}$ reactivation occurred in $13 \%$ of $\mathrm{HBsAg}$ positive patients who received antiviral prophylaxis during chemotherapy compared with $61 \%$ of patients who did not receive antiviral prophylaxis [3].

Univariate analysis in our cohort did not find HBV infection as a significant predictor of reduced overall survival. Our data suggest that patients who are HBsAg positive can have similar survival as HBsAg negative patients if they are given adequate antiviral prophylaxis. Therefore, approaches to prevent $\mathrm{HBV}$ reactivation are clinically important in HBV infected ALL patients undergoing chemotherapy.

HBV reactivation depends on the cancer type, host factors and types of therapy. HBV reactivation is particularly common in patients receiving chemotherapy for hematological malignancies, such as acute leukemia, because of the intensive chemotherapy regimens. The risk of HBV reactivation in patients receiving chemotherapy is a particular problem in endemic areas, especially among those who are HbsAg-positive. Rituximab is known to increase the risk of HBV reactivation, and a preventive strategy for HBV reactivation is required when rituximab is used. Rituximab was found to be useful in B-ALL [36]. A prospective study showed that rituximab in combination with an ALL chemotherapy protocol improved outcomes, including event-free survival, in younger adults with CD20-positive, Ph-negative ALL. There was longer event-free survival in the rituximab group than in the control group (65\% vs. 52\%) after a median follow-up of 30 months $(H R=0.66$; $p=0.04)$, attributed to lower rates of relapse of ALL [36].

$\mathrm{HBV}$ reactivation may be an exacerbation of a chronic hepatitis $B$ infection or recurrence of a previous infection. There are two major types of patients: those with a current infection (defined by the presence of $\mathrm{HBsAg}$ ), and those with occult $\mathrm{HBV}$ infection (defined by the presence of anti-HBC antibody). Patients with anti-HBc without $\mathrm{HBsAg}$ are considered to have resolved $\mathrm{HBV}$ infection, but they are still at risk of HBV reactivation and a serious or even fatal outcome during chemotherapy, particularly if receiving rituximab. Once the HBV infection has resolved, HBV DNA is rarely detected in peripheral blood, but trace amounts are often present in the liver, where the virus can be activated during immunosuppression [37].

Prophylactic antiviral therapy should be given to patients who are positive for HBsAg to prevent HBV reactivation. They should be given prophylactic antiviral treatment before initiation of chemotherapy or concurrently with chemotherapy, regardless of the level of HBV DNA. Antiviral prophylaxis is highly effective in preventing HBV reaction, with one reactivation prevented for every 3 patients treated [38]. However, a pre-emptive strategy, in which antiviral therapy is initiated once $\mathrm{HBV}$ reactivation is detected, is much less effective than prophylactic antiviral therapy in preventing hepatitis flares in $\mathrm{HBsAg}$-positive patients $[7,39,40]$. This is because of the time required for antiviral therapy to reduce viral load and control the disease (usually weeks to months), during which hepatic inflammation and necrosis can persist [41].

There is no standard strategy for the prevention of HBV reactivation in ALL patients with resolved HBV infection, and two possible strategies are available. One is to monitor HBV DNA regularly during treatment and initiate pre-emptive antiviral therapy as soon as HBV DNA becomes detectable. However, there is no consensus on the optimal interval of monitoring $[39,42]$, and HBV DNA monitoring is costly.

The other strategy is prophylactic antiviral therapy, which may be the preferred option during high-risk chemotherapy such as rituximab and anthracycline-based chemotherapy, and those without anti-HBs. This approach can effectively prevent $H B V$ reactivation without the inconvenience and cost of repeated HBV DNA monitoring [43]. Another potential advantage is that patients receive antiviral therapy early when there is minimal viremia, which is likely to optimize the efficacy of treatment, since antiviral agents tend to be less effective in patients with high viral titers.

We adopted a protocol of prophylactic antiviral therapy to prevent hepatitis flare due to HBV reactivation in our ALL patients receiving doxorubicin as part of a hyper-CVAD regimen based on data showing that prophylaxis is more effective than pre-emptive antiviral therapy in high-risk patients. Hyper-CVAD contains doxorubicin, which along with other anthracycline derivatives is considered to carry a moderate risk of $\mathrm{HBV}$ reactivation (1$10 \%$ risk) in patients with resolved HBV infection in the guideline published by the American College of Gastroenterology [44]. 
Another issue is the choice of antiviral prophylaxis. As the first oral antiviral agent to be used for the treatment of chronic HBV infection, lamivudine has been the most studied prophylactic agent. In randomized trials, lamivudine has proven to be effective in preventing $\mathrm{HBV}$ reactivation in patients receiving immunosuppressive therapies [40], and is well tolerated and safe. However, viral resistance can develop when lamivudine is used for more than 6 months because of the emergence of tyrosine-methionine-aspartic acid-aspartic acid (YMDD) mutations. As a result, newer agents such as entecavir or tenofovir, which are associated with a lower rate of resistance, are now preferred to lamivudine for the treatment of chronic HBV infection. In a prospective randomized study comparing lamivudine and entecavir in patients undergoing chemotherapy, the rate of HBV reactivation $(30 \%$ vs. $6.6 \%, p=0.001)$, HBV-related hepatitis (13.3\% vs. $0 \%, p=0.003)$, and chemotherapy disruption (18.3\% vs. $1.6 \%, p=0.002)$ were all significantly higher in the lamivudine than the entecavir group [9]. One of our patients on lamivudine prophylaxis developed hepatitis flare, indicating that lamivudine had been less effective in preventing HBV reactivation. Entecavir or tenofovir would be a better choice of antiviral prophylaxis. If the patient has renal impairment, entecavir would be more suitable as the choice of antiviral prophylaxis.

Limitations of our study include its retrospective design and relatively small sample size. In addition, some patients with profound thrombocytopenia did not undergo lumbar puncture at the time of initial diagnosis because of the bleeding risk, so we cannot rule out the possibility that some of these patients had CNS disease at baseline. Similarly, not all patients in our cohort were tested for anti-HBc because this test was not introduced as part of our routine viral testing protocol for ALL patients until 2010.

In conclusion, our study showed that hyperCVAD is effective, tolerable and easy to administer in adult ALL patients. It is an option for the treatment of ALL. The risk of hepatitis flare due to HBV reactivation can be reduced by administering antiviral prophylaxis in patients with HBV infection.

\section{Conflict of interest}

The authors declare no conflict of interest.

\section{References}

1. Kantarjian HM, O'Brien S, Smith TL, et al. Results of treatment with hyper-CVAD, a dose-intensive regimen, in adult acute lymphocytic leukemia. J Clin Oncol 2000; 18: 547-61.

2. Kantarjian H, Thomas D, O'Brien S, et al. Long-term follow-up results of hyperfractionated cyclophosphamide, vincristine, doxorubicin, and dexamethasone
(Hyper-CVAD), a dose-intensive regimen, in adult acute lymphocytic leukemia. Cancer 2004; 101: 2788-801.

3. Chen CY, Huang SY, Cheng A, et al. High risk of hepatitis $B$ reactivation among patients with acute myeloid leukemia. PLoS One 2015; 10: e0126037.

4. Niscola P, Del Principe MI, Maurillo L, et al. Fulminant $B$ hepatitis in a surface antigen-negative patient with B-cell chronic lymphocytic leukaemia after rituximab therapy. Leukemia 2005; 19: 1840-1.

5. Tsutsumi Y, Kawamura T, Saitoh S, et al. Hepatitis B virus reactivation in a case of non-Hodgkin's lymphoma treated with chemotherapy and rituximab: necessity of prophylaxis for hepatitis B virus reactivation in rituximab therapy. Leuk Lymphoma 2004; 45: 627-9.

6. Evens AM, Jovanovic BD, Su YC, et al. Rituximab-associated hepatitis B virus (HBV) reactivation in lymphoproliferative diseases: meta-analysis and examination of FDA safety reports. Ann Oncol 2011; 22: 1170-80.

7. Yeo W, Chan TC, Leung NW, et al. Hepatitis B virus reactivation in lymphoma patients with prior resolved hepatitis B undergoing anticancer therapy with or without rituximab. J Clin Oncol 2009; 27: 605-11.

8. Lim ST, Fei G, Quek R, et al. The relationship of hepatitis $B$ virus infection and non-Hodgkin's lymphoma and its impact on clinical characteristics and prognosis. Eur J Haematol 2007; 79: 132-7.

9. Huang $\mathrm{H}$, Li X, Zhu J, et al. Entecavir vs lamivudine for prevention of hepatitis $B$ virus reactivation among patients with untreated diffuse large B-cell lymphoma receiving $\mathrm{R}$-CHOP chemotherapy: a randomized clinical trial. JAMA 2014; 312: 2521-30.

10. Murphy SB, Bowman WP, Abromowitch M, et al. Results of treatment of advanced-stage Burkitt's lymphoma and B cell (Slg+) acute lymphoblastic leukemia with high-dose fractionated cyclophosphamide and coordinated high-dose methotrexate and cytarabine. J Clin Oncol 1986; 4: 1732-9.

11. Xu W, Li JY, Qian SX, et al. Outcome of treatment with Hyper-CVAD regimen in Chinese patients with acute lymphocytic leukemia. Leuk Res 2008; 32: 930-5.

12. Morris K, Weston H, Mollee P, Marlton P, Gill D, Kennedy G. Outcome of treatment of adult acute lymphoblastic leukemia with hyperfractionated cyclophosphamide, doxorubicin, vincristine, dexamethasone/methotrexate, cytarabine: results from an Australian population. Leuk Lymphoma 2011; 52: 85-91.

13. Abbasi S, Maleha F, Shobaki M. Acute lymphoblastic leukemia experience: epidemiology and outcome of two different regimens. Mediterr J Hematol Infect Dis 2013; 5: e2013024.

14. Buyukasik Y, Acar K, Kelkitli E, et al. Hyper-CVAD regimen in routine management of adult acute lymphoblastic leukemia: a retrospective multicenter study. Acta Haematol 2013; 130: 199-205.

15. Alacacioglu I, Medeni SS, Ozsan GH, et al. Is the BFM regimen feasible for the treatment of adult acute lymphoblastic leukemia? A retrospective analysis of the outcomes of BFM and hyper-CVAD chemotherapy in two centers. Chemotherapy 2014; 60: 219-23.

16. Portugal RD, Loureiro MM, Garnica M, Pulcheri W, Nucci $M$. Feasibility and outcome of the hyper-CVAD regimen in patients with adult acute lymphoblastic leukemia. Clin Lymphoma Myeloma Leuk 2015; 15: 52-7.

17. Bassan R, Hoelzer D. Modern therapy of acute lymphoblastic leukemia. J Clin Oncol 2011; 29: 532-43.

18. Abdelsalam L, Elshobaky MA, El-araby RE, et al. Expression of beclin-1 and apoptosis-related genes in child- 
hood acute lymphoblastic leukemia. Arch Med Sci Civil Dis 2017; 2: e168-73.

19. Noda NN, Inagaki F. Mechanisms of autophagy. Annu Rev Biophys 2015; 44: 101-22.

20. Thomas DA, O'Brien S, Faderl S, et al. Chemoimmunotherapy with a modified hyper-CVAD and rituximab regimen improves outcome in de novo Philadelphia chromosome-negative precursor B-lineage acute lymphoblastic leukemia. J Clin Oncol 2010; 28: 3880-9.

21. Tanguy-Schmidt A, Rousselot $P$, Chalandon $Y$, et al. Long-term follow up of the imatinib GRAAPH-2003 study in newly diagnosed patients with de novo Philadelphia chromosome-positive acute lymphoblastic leukemia: a GRAALL study. Biol Blood Marrow Transplant 2013; 19: 150-5.

22. Bassan R, Rossi G, Pogliani EM, et al. Chemotherapy-phased imatinib pulses improve long-term outcome of adult patients with Philadelphia chromosome-positive acute lymphoblastic leukemia: Northern Italy Leukemia Group protocol 09/00. J Clin Oncol 2010; 28: 3644-52.

23. Kantarjian H, Shah NP, Hochhaus A, et al. Dasatinib versus imatinib in newly diagnosed chronic-phase chronic myeloid leukemia. N Engl J Med 2010; 362: 2260-70.

24. Saglio G, Kim DW, Issaragrisil S, et al.; ENESTnd Investigators. Nilotinib versus imatinib for newly diagnosed chronic myeloid leukemia. N Engl J Med 2010; 362: 2251-9.

25. Kantarjian HM, Shah NP, Cortes JE, et al. Dasatinib or imatinib in newly diagnosed chronic-phase chronic myeloid leukemia: 2-year follow-up from a randomized phase 3 trial (DASISION). Blood 2012; 119: 1123-9.

26. Hu Y, Liu Y, Pelletier S, et al. Requirement of SRC kinases Lyn, Hck and Fgr for BCR-ABL1-induced B-lymphoblastic leukemia but not chronic myeloid leukemia. Nat Genet 2004; 36: 453-61.

27. Ravandi F, O'Brien SM, Cortes JE, et al. Long-term follow-up of a phase 2 study of chemotherapy plus dasatinib for the initial treatment of patients with Philadelphia chromosome-positive acute lymphoblastic leukemia. Cancer 2015; 121: 4158-64.

28. Sasaki K, Jabbour EJ, Ravandi F, et al. Hyper-CVAD plus ponatinib versus hyper-CVAD plus dasatinib as frontline therapy for patients with Philadelphia chromosome-positive acute lymphoblastic leukemia: a propensity score analysis. Cancer 2016; 122: 3650-6.

29. Saadeh SS, Litzow MR. Hematopoietic stem cell transplant in adults with acute lymphoblastic leukemia: the present state. Expert Rev Hematol 2018; 11: 195-207.

30. Fielding AK, Rowe JM, Buck G, et al. UKALLXII/ECOG2993: addition of imatinib to a standard treatment regimen enhances long-term outcomes in Philadelphia positive acute lymphoblastic leukemia. Blood 2014; 123: 843-50.

31. Chan AO, Yuen MF, Lam CM, Fong CY, Wong BC, Lai CL. Prevalence and characteristics of familial hepatocellular carcinoma caused by chronic hepatitis B infection in Hong Kong. Aliment Pharmacol Ther 2004; 19: 401-6.

32. Engels EA, Cho ER, Jee SH. Hepatitis B virus infection and risk of non-Hodgkin lymphoma in South Korea: a cohort study. Lancet Oncol 2010; 11: 827-34.

33. Law MF, Lai HK, Chan HN, et al. The impact of hepatitis $B$ virus (HBV) infection on clinical outcomes of patients with diffuse large B-cell lymphoma. Eur J Cancer Care 2015; 24: 117-24.

34. Natoli G, Avantaggiati ML, Chirillo P, et al. Ras- and Raf dependent activation of c-jun transcriptional activity by the hepatitis B virus transactivator pX. Oncogene 1994; 9: 2837-43.

35. Wang XW, Gibson MK, Vermeulen W, et al. Abrogation of p53-induced apoptosis by the hepatitis $B$ virus $X$ gene. Cancer Res 1995; 55: 6012-6.

36. Maury S, Chevret S, Thomas X, et al.; for GRAALL. Rituximab in B-lineage adult acute lymphoblastic leukemia. N Engl J Med 2016; 375: 1044-53.

37. Law MF, Ho R, Cheung CK, et al. Prevention and management of hepatitis $B$ virus reactivation in patients with hematological malignancies treated with anticancer therapy. World J Gastroenterol 2016; 22: 6484-500.

38. Lau GK, Yiu HH, Fong DY, et al. Early is superior to deferred preemptive lamivudine therapy for hepatitis B patients undergoing chemotherapy. Gastroenterology 2003; 125: 1742-9.

39. Hsu C, Tsou HH, Lin SJ, et al.; Taiwan Cooperative Oncology Group. Chemotherapy-induced hepatitis B reactivation in lymphoma patients with resolved HBV infection: a prospective study. Hepatology 2014; 59: 2092-100.

40. Hsu C, Hsiung CA, Su IJ, et al. A revisit of prophylactic lamivudine for chemotherapy-associated hepatitis B reactivation in non-Hodgkin's lymphoma: a randomized trial. Hepatology 2008; 47: 844-53.

41. Di Bisceglie AM, Lok AS, Martin P, Terrault N, Perrillo RP, Hoofnagle JH. Recent US Food and Drug Administration warnings on hepatitis $B$ reactivation with immune-suppressing and anticancer drugs: just the tip of the iceberg? Hepatology 2015; 61: 703-11.

42. Fukushima N, Mizuta T, Tanaka M, et al. Retrospective and prospective studies of hepatitis $B$ virus reactivation in malignant lymphoma with occult HBV carrier. Ann Oncol 2009; 20: 2013-7.

43. Huang YH, Hsiao LT, Hong YC, et al. Randomized con trolled trial of entecavir prophylaxis for rituximab-as sociated hepatitis $B$ virus reactivation in patients with lymphoma and resolved hepatitis B. J Clin Oncol 2013; 31: 2765-72.

44. Perrillo RP, Gish R, Falck-Ytter YT. American Gastroenterological Association Institute technical review on prevention and treatment of hepatitis $B$ virus reactivation during immunosuppressive drug therapy. Gastroenterol ogy 2015; 148: 221-4.e3 